\title{
Wissenschaftskommunikation und Social Media: Neue Akteure, Polarisierung und Vertrauen
}

\author{
Carsten Könneker
}

Dieser Beitrag ${ }^{1}$ analysiert, wie soziale Medien die externe Wissenschaftskommunikation verändern: z. B. durch eine Vielzahl Neuer Akteure, von denen einzelne massenmediale Wirkungen erzielen, sowie durch neue Aufmerksamkeits- und Traffic-Ströme für wissenschaftsjournalistische Portale mit Rückwirkungen auf redaktionelle Strategien und Organisationsstrukturen. Auch Forschungseinrichtungen, wissenschaftsfördernde Institutionen und andere Organisationen sind mit eigenen Informations- und Meinungsangeboten zu Wissenschaftsthemen in sozialen Medien aktiv ebenso wie Akteure mit antiaufklärerischer Agenda und Verfechter von Verschwörungsmythen. Dies provoziert die Frage, ob wissenschaftsskeptische Inhalte in sozialen Netzwerken, jenseits des Filters und der Moderation durch traditionelle Gatekeeper des Mediensystems, in besonderer Weise florieren können. Denn auch zu Wissenschaftsthemen werden Diskussionen in Social Media oft emotional geführt, Communitys mobilisieren und radikalisieren sich. Als Erklärung für derlei Polarisierungsphänomene werden mehrere psychologische Effekte angeführt, welche gleichfalls in Offline-Kontexten virulent sind, unter den Bedingungen sozialer Netzwerke

\footnotetext{
${ }^{1}$ Rainer Bromme (Münster) und Arnd Florack (Wien) sei herzlich für die kritische Durchsicht des Manuskripts und wichtige Anregungen gedankt.

C. Könneker $(\bowtie)$

Spektrum der Wissenschaft, Heidelberg, Deutschland

E-Mail: koenneker@spektum.de
} 
jedoch größere Wirkungen entfalten können. Dies führt dazu, dass sich vorhandene (wissenschaftsskeptische) Einstellungen in Social-Media-Diskursen stärker verfestigen und radikalisieren können. Eine forcierende Rolle kommt hierbei verschiedenen technologischen Effekten sowie der (faktischen oder vermeintlichen) Anonymität in Online-Diskussionen zu. Ausgangs diskutiert der Beitrag, wie in digital vernetzten Öffentlichkeiten gesellschaftliches Vertrauen in Wissenschaft gesichert bzw. (wieder-)hergestellt werden kann. Wichtig erscheinen hier einerseits Aspekte der praktischen Wissenschaftskommunikation wie mehr authentische Einblicke in die Motive und Methoden von Forschenden sowie andererseits verbesserte institutionelle Rahmenbedingungen wie mehr Wertschätzung für ein entsprechendes Engagement von Forschenden sowie ein Ausbau einschlägiger praxisorientierter Lehrangebote.

\section{Modell der Wissenskommunikation und Neue Akteure}

Dieser Beitrag vertritt einen weiten Begriff von Wissenschaftskommunikation, der insbesondere auch den Wissenschaftsjournalismus eingedenk seiner spezifischen Besonderheiten und Funktionen umfasst. Hierzu werden in der Literatur verschiedene Modelle vertreten, die teils jedoch problematisch erscheinen, weil sie nahelegen, fremdvermittelte externe Wissenschaftskommunikation mit Wissenschaftsjournalismus gleichzusetzen (z. B. acatech et al. 2017, S. 21; Dogruel und Beck 2017, S. 142; Schäfer 2017; vgl. Hagenhoff et al. 2007). Tatsächlich macht der Wissenschaftsjournalismus jedoch nur einen - wenn auch nach wie vor bedeutenden und für demokratische Gesellschaften eminent wichtigen - Teil fremdvermittelter externer Wissenschaftskommunikation aus. Doch auch Politiker, die sich zu Wissenschaftsthemen äußern, oder Schriftsteller, die solche in ihren Werken aufgreifen und reflektieren, ${ }^{2}$ wie auch Teile der Popkultur ${ }^{3}$ praktizieren fremdvermittelte externe Wissenschaftskommunikation, um drei konventionelle, teils traditionsreiche ${ }^{4}$ Beispiele nur anzureißen (Abb. 1). Unter den Bedingungen von Social Media entstand jedoch eine - rasant gewachsene und äußerst heterogene - Gruppe innerhalb der weiteren Akteure, die ich „Neue Akteure der Wissenschaftskommunikation“ nennen möchte: Personen

\footnotetext{
${ }^{2}$ Für physikalische Diskurse vgl. z. B. Özelt (2018).

${ }^{3}$ Für die Darstellung der Lebenswissenschaften in der Popkultur vgl. Allgaier (2019).

${ }^{4}$ Man denke z. B. an das Genre des Wissenschaftsromans, für dessen Tradition in der deutschsprachigen Literatur Werke wie Goethes Die Wahlverwandtschaften (1809) oder Hermann Brochs Die Unbekannte Größe (1933) stehen.
} 


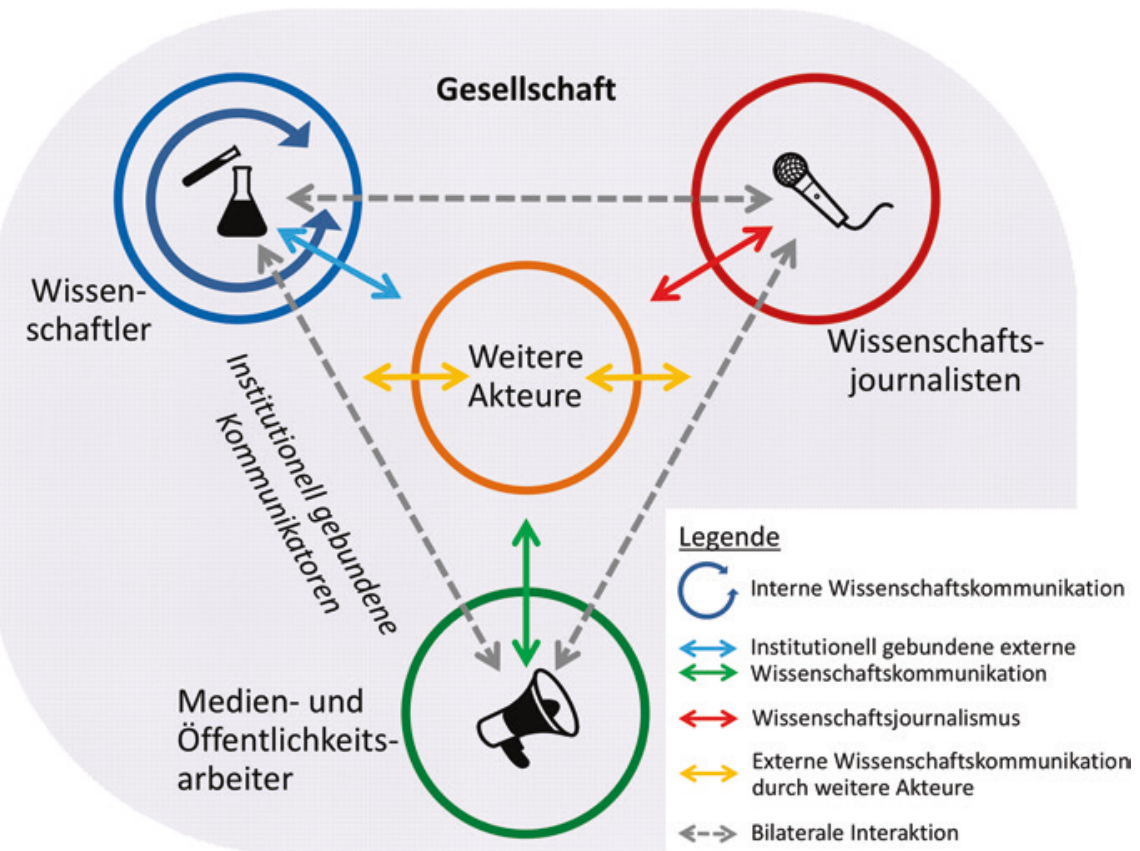

Abb. 1 Modell der Wissenschaftskommunikation nach Akteuren (nach Könneker 2016). Außer Wissenschaftsjournalistinnen und -journalisten praktizieren diverse weitere Akteure fremdvermittelte externe Wissenschaftskommunikation

einerseits ohne Affiliation zu wissenschaftlichen Institutionen - und damit fremdvermittelnd agierend - sowie andererseits ohne Erfüllung redaktioneller Standards wie beispielsweise systematische Überprüfung von Quellen und Vier-Augen-Prinzip - und damit nicht-journalistisch agierend. Sie erweitern das Feld der externen Wissenschaftskommunikation in erheblichem Ausmaß. Ermöglicht wird dies durch die radikal geschrumpfte Zutrittsbarrieren zum (massen-)medialen Betrieb durch die sozialen Netzwerke.

Dass einzelne dieser Neuen Akteure der externen Wissenschaftskommunikation massenmediale Wirkungen erzielen können, zeigen im englischsprachigen Social Web u. a. Beispiele von Youtubern wie Derek Muller, dessen 2010 eröffneter Kanal „Veritasium“ bis Herbst 2018 bei fast einer halben Milliarde Aufrufen ${ }^{5}$ beinahe 5 Millionen Abonnenten erreichte, ${ }^{6}$

\footnotetext{
${ }^{5}$ Laut Kanalinfo auf YouTube waren es am 12. Oktober 2018 458.607.244 Aufrufe (https://www.youtube.com/user/1veritasium/about - Zugegriffen: 12. Oktober 2018).

${ }^{6}$ Laut Übersicht des Kanals auf YouTube waren es am 12. Oktober 2018 4.964.175 Abonnenten (https://www.youtube.com/user/1veritasium/featured - Zugegriffen: 12. Oktober 2018). Zur Bedeutung von YouTube für die externe Wissenschaftskommunikation siehe Allgaier (2018).
} 
Millionen Visits

Anteil Social Traffic

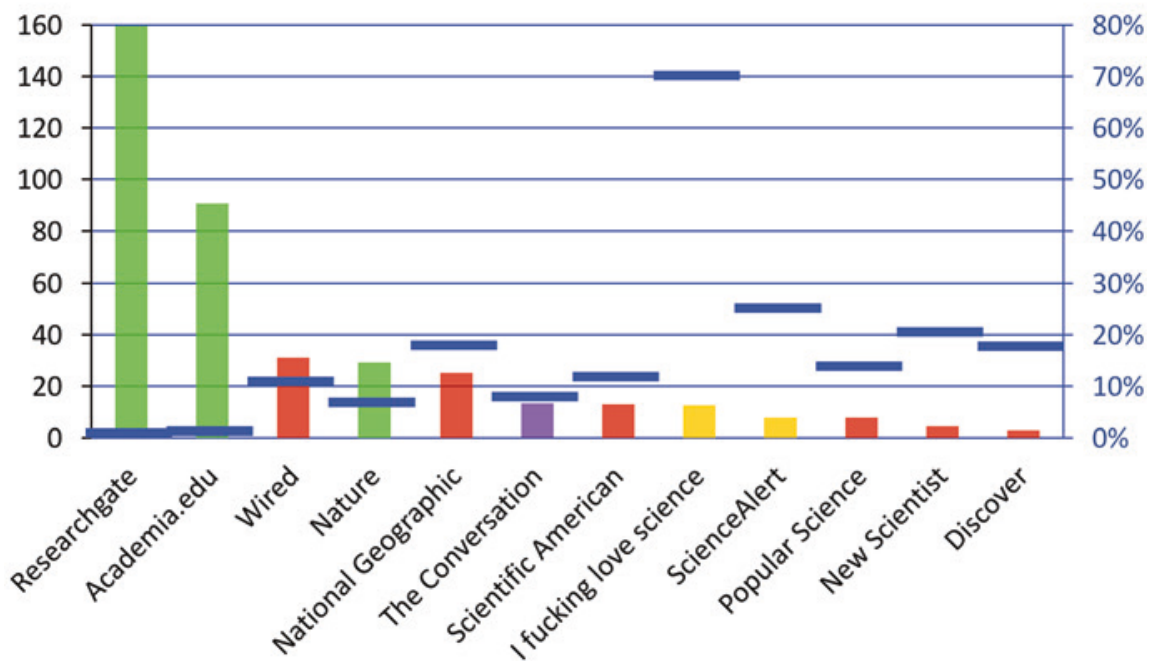

Abb. 2 Zugriffszahlen (in Visits) ausgewählter englischsprachiger Wissenschaftsportale im Monat November 2018 unter Wiedergabe des jeweiligen Anteils an Social Traffic (Daten per Similarweb abgeschätzt). Neben den in grün dargestellten Portalen der internen Wissenschaftskommunikation ResearchGate, Academia.edu und Nature sind in rot Portale journalistischer Provenienz aufgeführt, die jeweils eine populärwissenschaftliche Zeitschrift zum Ursprung haben, nämlich Wired, National Geographic, Scientific American, Popular Science, New Scientist sowie das Discover Magazine. In diese Gruppe stoßen mit vergleichbaren Reichweiten neuere Portale wie The Conversation, IFLScience und ScienceAlert vor, die andere Ursprünge haben (gelb dargestellt: Portale von Neuen Akteuren der Wissenschaftskommunikation, die jeweils aus einer Facebook-Seite hervorgegangen sind; lila: weitere)

oder Hashem Al-Ghaili, dessen Wissenschaftsvideos speziell auf Facebook, aber auch auf YouTube und in weiteren Netzwerken zum selben Zeitpunkt sogar noch reichweitenstärker waren. ${ }^{7}$ Zuerst als Facebook-Seite entstand 2012 zudem das Angebot „I fucking love science“8. Elise Andrew, die Urheberin, postete dort mit einer auch dem Journalismus zwar nicht fremden, dessen Funktion im Kern jedoch zuwiderlaufenden Attitüde der Bewunderung für (skurrile) wissenschaftliche Erkenntnisse und erzielte damit schnell erhebliche Resonanz. Erst daraufhin launchte sie mit IFLScience eine eigene Plattform außerhalb von Facebook; ${ }^{9}$ der Ursprung dieses Portals ist indes noch immer

\footnotetext{
${ }^{7} \mathrm{Al}-$ Ghailis reichweitenstärkstes Angebot ist seine „Science Nature Page“ auf Facebook (https://www. facebook.com/ScienceNaturePage) mit 30,4 Millionen Abonnenten bei mehr als 10 Milliarden einzelnen Seitenaufrufen - Zugegriffen: 04. Dezember 2018.

${ }^{8}$ https://de-de.facebook.com/IFeakingLoveScience; Anzahl der Abonnenten: 24,8 Millionen Zugegriffen: 04. Dezember 2018.

${ }^{9}$ https://iflscience.com/ - Zugegriffen: 04. Dezember 2018.
} 


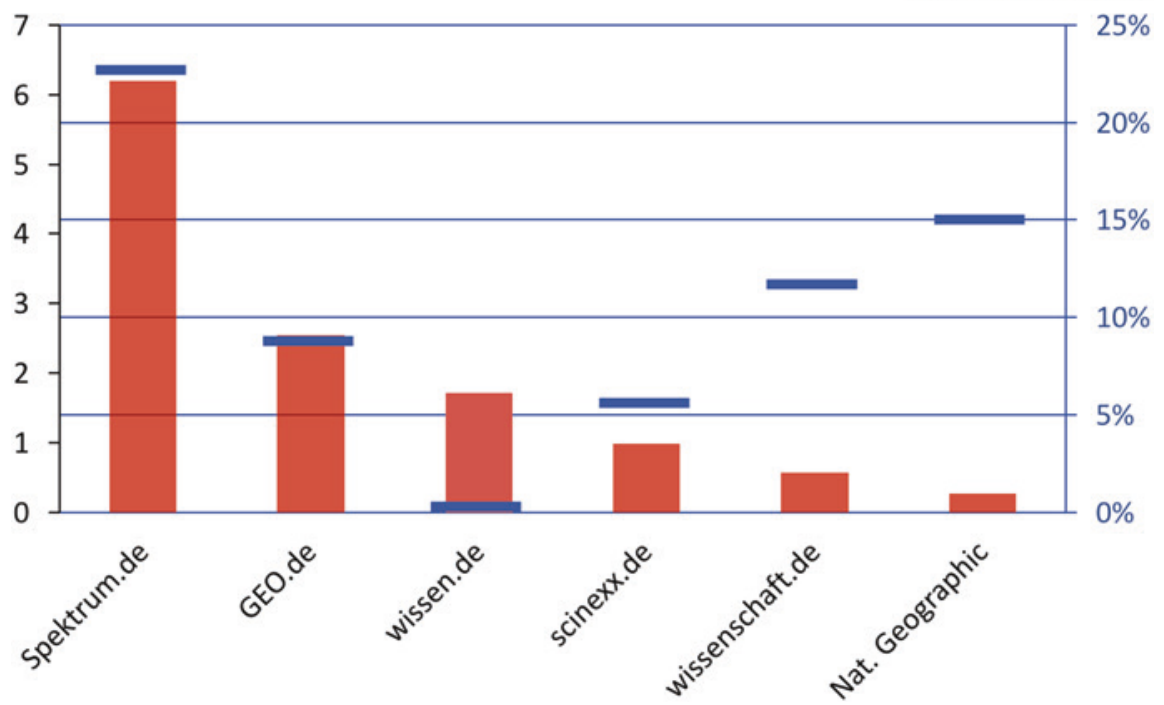

Abb. 3 Zugriffszahlen (in Visits) ausgewählter deutschsprachiger Wissens- und Wissenschaftsportale im Monat September 2018 unter Wiedergabe des jeweiligen Anteils an Social Traffic (Daten per Similarweb abgeschätzt). (Quelle: Autor)

an seinem vergleichsweise hohen Anteil an Social Traffic zu erkennen (Abb. 2). So stammten von den abgeschätzten 12.810 .000 Visits von IFLScience im November 2018 70,2 Prozent aus sozialen Netzwerken (und hier fast ausschließlich von Facebook); im Juli 2018 waren es sogar 85,1 Prozent von 31.600.000 Visits.

Als traditionelle Anbieter populärwissenschaftlicher Inhalte, die ihre Geschäftsmodelle immer weiter in Richtung digitaler Angebote weiterentwickeln, stehen Verlage vor der Frage, wie viel Ressourcen sie mit welcher Zielsetzung in das Bespielen eigener Social-Media-Präsenzen investieren (Neuberger 2014); etliche Redaktionen wurden so um das Aufgabenprofil des Community Managements erweitert. Auch bei den einschlägigen deutschsprachigen Portalen spielt die Reichweitengewinnung über soziale Netzwerke teils eine strategische Rolle (Abb. 3). Während der Anteil der über soziale Medien generierten Visits der Portale von Spektrum der Wissenschaft sowie der Gruner und Jahr-Titel GEO und National Geographic Deutschland ähnlich wie die der meisten journalistischen englischsprachigen Portale aus Abb. 2 über das Jahr 2018 meist im Bereich zwischen 10 und 20 Prozent lagen, ${ }^{10}$

\footnotetext{
${ }^{10} \mathrm{Als}$ Beispiel sind die Zahlen des Monats September 2018 aufgeführt.
} 
rangierten etwa die von der Konradin-Verlagsgruppe betriebenen Portale wissen.de sowie wissenschaft.de (die bild der wissenschaft zugehörige Plattform) in den meisten Monaten unterhalb von 5 Prozent, d. h., sie bezogen umgekehrt mehr Traffic-Anteile über Suchmaschinen, Verlinkungen (Referrals) und Direktzugriffe (Directs).

\section{Social Media-Nutzung und Wissenschaft auf Facebook}

Um die Bedeutung von Social Media für die externe Wissenschaftskommunikation auch im deutschsprachigen Internet genauer fassen zu können, ist es wichtig, sich vor Augen zu führen, in welchen sozialen Netzwerken die Deutschen wie aktiv sind. Laut der bevölkerungsrepräsentativen ARD/ZDF-Onlinestudie (Frees und Koch 2018) waren 201831 Prozent der Deutschen ab 14 Jahren regelmäßig, d. h. wöchentlich oder häufiger, auf Facebook aktiv. Stärker noch, nämlich zu 39 Prozent, nutzten sie Videoportale wie YouTube regelmäßig. Hier ist - anders als bei Facebook, wo der Zuspruch hiesiger Nutzerinnen und Nutzer seit 2016 vor allem bei den unter 30-Jährigen sinkt - im Jahresvergleich ein dynamisches Wachstum zu registrieren, ähnlich wie bei den insgesamt weniger stark, dafür aber bevorzugt von jungen Nutzerinnen und Nutzern frequentierten Netzwerken Instagram und Snapchat (Abb. 4). Twitter ist mit insgesamt 4 Prozent regelmäßiger Nutzung in Deutschland ein vergleichsweise kleines soziales Netzwerk, allerdings mit einer hohen Dichte an Entscheidungsträgern aus Politik und Medien unter seinen Mitgliedern. Knapp die Hälfte der Deutschen nutzt täglich das Smartphone als Zugang zum Internet, gefolgt von stationärem Rechner, Laptop sowie Tablet. Unter den jüngeren Nutzern ist die tägliche Webnutzung per Smartphone mit 86 Prozent noch erheblich stärker ausgeprägt (Abb. 5).

Auf Facebook findet sich ein äußert mannigfaltiges Angebot deutschsprachiger Beiträge zur Wissenschaftskommunikation. Traditionelle Medienmarken aus dem öffentlich-rechtlichen wie dem privatwirtschaftlichen Bereich, insbesondere populärwissenschaftliche TV-Sendungen und Zeitschriften, sind ebenso vertreten wie beispielsweise Hochschulen, außeruniversitäre Forschungseinrichtungen, Wissenschaftsorganisationen, Akademien, wissenschaftsfördernde Stiftungen, Museen und Ministerien. Darüber hinaus sind auf Facebook namhafte Publizisten wie Eckart von Hirschhausen oder 


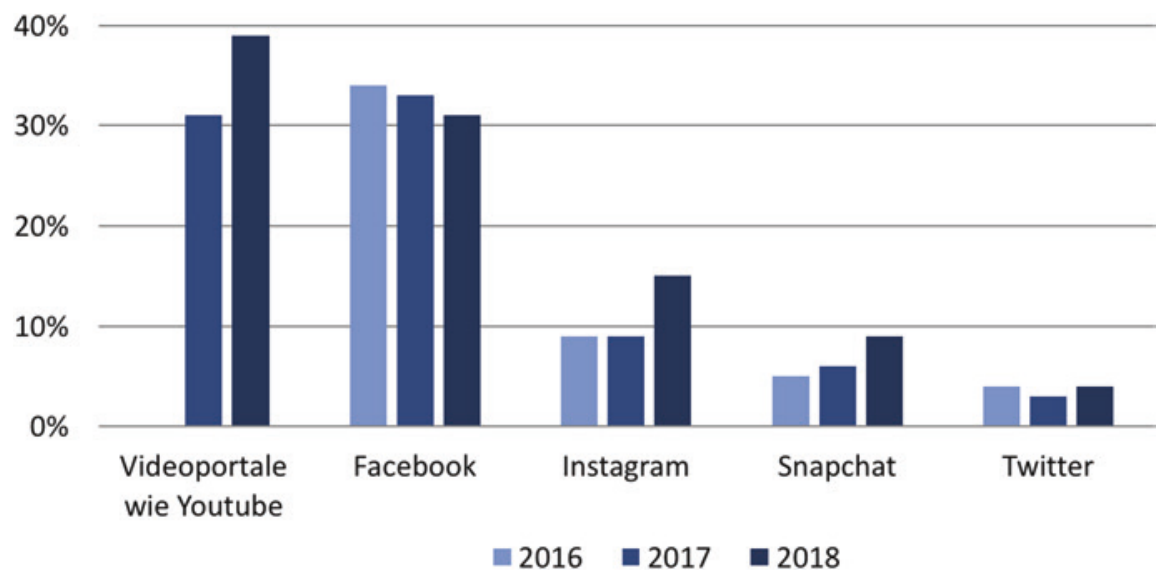

Abb. 4 Entwicklung der Anzahl regelmäßiger (d. h. mindestens wöchentlich aktiver) Nutzerinnen und Nutzer ausgewählter sozialer Netzwerke in Deutschland gemäß ARD/ZDF-Onlinestudie 2016 bis 2018. Die Angaben zu den Videoportalen für 2016 fehlen, da erst ab 2017 entsprechend erhoben wurde. (Quelle: Autor)

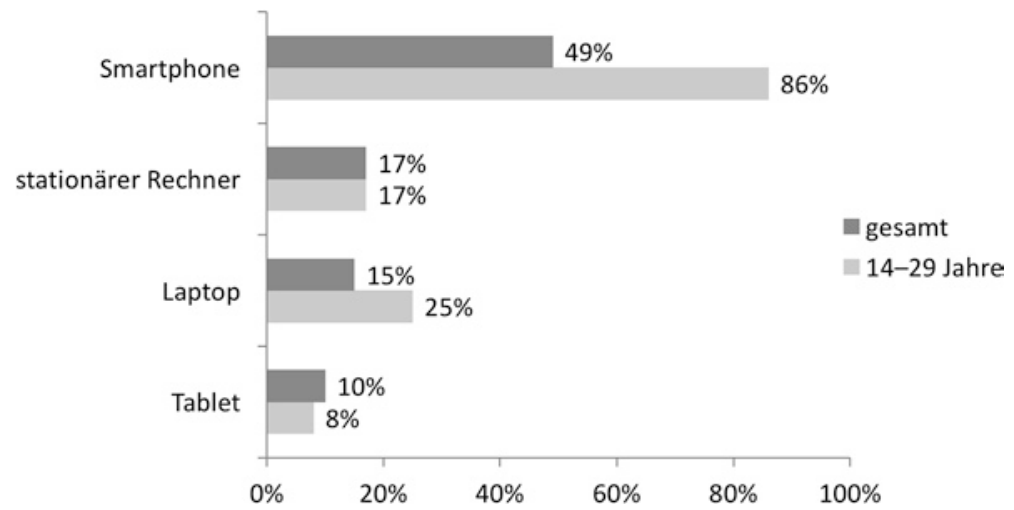

Abb. 5 Regelmäßige Gerätenutzung („Nutzung gestern“) für den Onlinezugang gemäß ARD/ZDF-Onlinestudie 2018, unterteilt in Gesamtbevölkerung Deutschland sowie jüngere Nutzerinnen und Nutzer (14 bis 29 Jahre). (Quelle: Autor)

Ranga Yogeshwar, aber auch - der Breite der Bevölkerung teils eher weniger bekannte - Wissenschaftsyoutuber wie Borja Schwember („Dr. Allwissend“) ${ }^{11}$ oder Philip Häusser ${ }^{12}$ vertreten (Abb. 6).

\footnotetext{
${ }^{11}$ https://www.youtube.com/user/doktorallwissend - Zugegriffen: 29. Dezember 2018.

${ }^{12}$ Häusser produzierte bis November 2018 für den eigenen YouTube-Kanal „Phil’s Physics“. Seither ist dieses Angebot in dem erweiterten „Breaking Lab“ aufgegangen: https://www.youtube.com/channel/ UCE2hJ9CYR57BYhk3TjGVG6w - Zugegriffen: 29. Dezember 2018.
} 


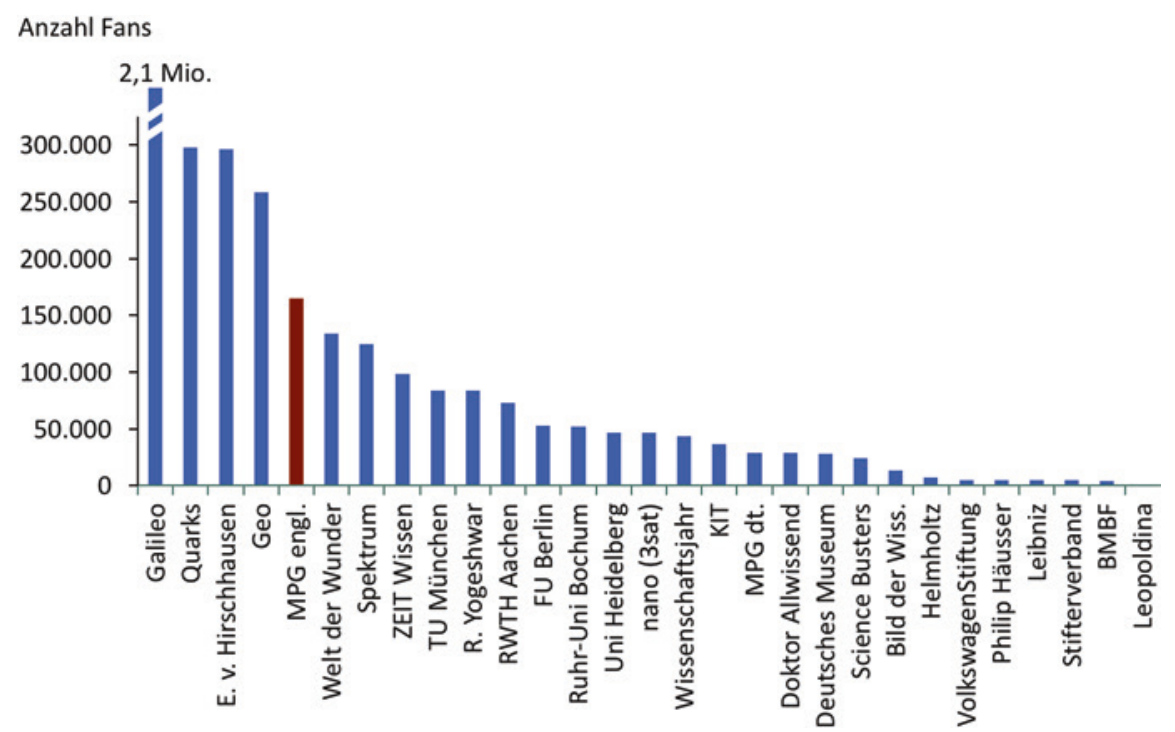

Abb. 6 Deutschsprachige Angebote der externen Wissenschaftskommunikation (Auswahl) nach Anzahl der Facebook-Fans („Gefällt mir“) - Stand: 29. Oktober 2018. In rot dargestellt ist das englischsprachige Angebot der Max-Planck-Gesellschaft. (Quelle: Autor)

Das heterogene Portfolio ist durch Angebote weiterer Akteure zu ergänzen, die dezidiert wissenschaftsskeptische oder antiaufklärerische Inhalte propagieren. Ein Beispiel hierfür ist die Facebook-Präsenz der „German Chemtrail Watchers"13, welche den Verschwörungsglauben verbreitet, dass die Kondensstreifen von Flugzeugen nicht auf Abgase zurückgehen, sondern auf absichtlich - etwa regierungsseits - ausgebrachte Chemikalien. Gemessen an ihren Fanzahlen rangierte die Seite 2018 in etwa gleichauf mit jenen der Helmholtz-Gemeinschaft oder der VolkswagenStiftung. ${ }^{14}$ Als weiteres Beispiel sei die Facebook-Seite der "Flat Earth Society “ angeführt, ${ }^{15}$ welche die Auffassung propagiert, die Erde habe keine Kugelgestalt, sondern sei empirisch belegbar flach. Diese englischsprachige Seite zählte 2018 mehr Fans als die englischsprachige Facebook-Seite der Max-Planck-Gesellschaft. ${ }^{16}$

\footnotetext{
${ }^{13}$ https://de-de.facebook.com/German-Chemtrail-Watchers-113843298718096/ - Zugegriffen: 13. Dezember 2018.

${ }^{14}$ Anzahl der Facebook-Fans (Stand: 29. August 2018): 7111 Helmholtz-Gemeinschaft; 6626 German Chemtrail Watchers; 5215 VolkswagenStiftung.

${ }^{15}$ https://www.facebook.com/FlatEarthToday - Zugegriffen: 13. Dezember 2018.

${ }^{16} 167.129$ Facebook-Fans gegenüber 164.781 (Stand: 29. August 2018).
} 
Die Reichweitenerfolge inhaltlich höchst fragwürdiger Facebook-Seiten wie der genannten erscheinen insbesondere vor dem Hintergrund brisant, dass 54 Prozent der deutschen Facebook-Nutzer nach eigener Einschätzung Nachrichten des Qualitätsjournalismus auf dem sozialen Netzwerk nicht von Falschmeldungen unterscheiden können, da sich die Darstellungen stark ähneln (Edelman Trust Barometer 2018).

Die für Rezipienten nur unzureichend nach Quellen differenzierende Darstellung von Inhalten stellt indes nur eines von mehreren gravierenden Problemen dar. Ein anderes ist die von Algorithmen gesteuerte personalisierte Ausspielung der Inhalte. Das Geschäftsmodell sozialer Netzwerke wie Facebook oder YouTube gründet darauf, Nutzer möglichst intensiv zu involvieren, d. h. zu möglichst vielen Interaktionen zu motivieren und sie dabei möglichst lange auf der jeweiligen Plattform zu halten. Denn die Vielzahl so gewonnener Daten über die individuellen Präferenzen der Nutzerinnen und Nutzer gestattet es den Netzwerk-Betreibern, individualisiert Werbung auszuspielen. Die auf KI-gestützten Big-Data-Analysen basierenden Facebook-Algorithmen etwa erzeugen für jede einzelne Nutzerin, jeden einzelnen Nutzer ein Modell sämtlicher persönlicher Präferenzen; auf Grundlage des Modells der realen Person werden dann solche Inhalte ausgespielt, die diese mit größtmöglicher Wahrscheinlichkeit zu weiteren Interaktionen innerhalb des Netzwerks veranlassen.

Derlei Mechanismen fördern diverse Community-Effekte: Personen mit ähnlichen Einstellungen können sich in sozialen Netzwerken über räumliche und zeitliche Grenzen hinweg identifizieren, miteinander austauschen und anlassbezogen mitunter auch sehr kurzfristig - gegenseitig mobilisieren. Plakativ formuliert: Wenn Tausende Menschen in Deutschland den Verdacht hegen, Regierungen ließen zur Eindämmung des Bevölkerungswachstums, zur Manipulation von Gedanken oder für militärische Zwecke gezielt Gifte von Flugzeugen in die Atmosphäre emittieren, dann sind die einzelnen Anhänger dieses Verschwörungsglaubens vergleichsweise isoliert, wenn sie ihre Sichtweise offensiv an den Stammtischen ihrer jeweiligen Wohnorte ausbreiten. Das Web 2.0 indes gestattet es ihnen - wie auch allen anderen Personen, die gleiche Ansichten, Einstellungen und Interessen pflegen -, einander zu finden, sich in ihren Auffassungen gegenseitig zu bestärken, ja sich gemeinsam zu erhitzen. Das macht soziale Netzwerke zu einem Motor gesellschaftlicher Polarisierung - auch, aber längst nicht nur in Bezug auf Wissenschaftsthemen. 


\section{Psychologische Effekte der Kommunikation und Meinungsbildung}

Um die Wucht der Entwicklung genauer zu fassen, werden im Folgenden sieben psychologische Effekte näher erläutert, von denen sechs - auch unabhängig von digitalen Diskursen - bereits in der Literatur beschrieben wurden und als etabliert gelten; der siebte wird im Sinne eines Vorschlags eingebracht. Die übergeordnete These hierbei lautet: Wann immer Menschen Informationen oder Meinungsbeiträge auswählen, untereinander austauschen oder diskutieren, unterliegen sie diesen Effekten in Abhängigkeit etwa von individuellen Persönlichkeitseigenschaften mehr oder weniger stark. Unter den Bedingungen sozialer Netzwerke können die Wirkungen jedoch ungleich größer ausfallen, da andere, auch technologische Effekte, sie verstärken.

Confirmation Bias Hiermit bezeichnen Psychologen den Umstand, dass wir Informationen vorzugsweise so auswählen, deuten und erinnern, dass sie unsere bereits bestehende Sichtweise stützen. Das ebenfalls als Bestätigungsfehler bezeichnete Phänomen wurde bereits für Social-Media-Diskussionen untersucht. So zeigten Anhänger von Verschwörungstheorien und Wissenschaftsinteressierte - bei unterschiedlicher Ausbreitungsdynamik - ähnliche Nutzungsgewohnheiten auf Facebook, wenn sie Neuigkeiten teilten (Del Vicario et al. 2016); in beiden Domänen bildeten sich zudem jeweils homogene, polarisierte Communitys (Echokammern) heraus.

Partisan Bias Dieser Effekt bezeichnet eine Wahrnehmungsverzerrung zugunsten der eigenen Bezugsgruppe. Informationen aus gegnerischen Communitys werten wir ab, inkohärente oder fehlerhafte Informationen aus der eigenen bewerten wir hingegen milder. Als Illustration mag ein Nutzerkommentar auf der Facebook-Seite von Spektrum der Wissenschaft dienen, der sich auf einen dort geposteten redaktionellen Beitrag zweier Wissenschaftler zum Thema „Verschwörungstheorien“ bezieht:

Diese Professoren labern aber auch ein Fachchinesisch. Klar ist auf jeden Fall, diese Professoren verdienen ihr Geld mit dem was sie da raus lassen. Was aber mit den Verschwörungstheorien ist, bleibt im Dunkeln. Sinnloses Geschwafel von überflüssigen Wissenschaftlern. ${ }^{17}$

\footnotetext{
${ }^{17}$ https://www.facebook.com/spektrumverlag, 12. August 2017 - Zugegriffen: 13. August 2017.
} 
Motivated Reasoning Ständig suchen, verwenden und bewerten wir Informationen. Dies tun wir allerdings nicht „neutral“. Vielmehr werden Schlüsse, die wir aus den Informationen ziehen, ständig durch innere Beweggründe verzerrt (Kunda 1990). In diesem allgemeinen Sinne können Confirmation Bias und Partisan Bias als Varianten des Motivated Reasoning angesehen werden. In einem engeren Sinne wird unter Motivated Reasoning jedoch eine Form der Emotionsregulation bei Entscheidungen verstanden, die auf das Vermeiden kognitiver Dissonanz abzielt. Kognitive Dissonanz entsteht, wenn wir gleichzeitig einander widersprechende Wertvorstellungen oder inhaltlich unvereinbare Annahmen zu vereinen suchen. Um den damit einhergehenden psychischen Stress zu regulieren, reagieren wir auf solche Informationen positiv, die stützen, was wir zuvor schon glaubten oder fühlten (Frimer et al. 2017). Zur Veranschaulichung mag dieser Nutzerkommentar zu einem von der Organisation „Ärzte gegen Tierversuche“ auf deren Facebook-Seite geposteten Video dienen, in dem sich ein Notfallmediziner kritisch gegenüber Experimenten an Tieren geäußert hatte:

Vielen vielen Dank für Eure Arbeit und diesen aufschlussreichen Beitrag, er bestätigt das, was ich schon lange gefühlt habe, wenn ich von Tierversuchen gelesen oder gehört habe. Ich unterstütze Euch von ganzem Herzen und aus tiefster Überzeugung. Es ist ein Wahnsinn was in manchen Laboren passiert. ${ }^{18}$

Backfire-Effekt Hierunter fassen Psychologen den Umstand, dass Versuche, inhaltlich falsche Annahmen durch Bereitstellung geprüfter Fakten von neutraler Seite auszuräumen, mitunter bewirken, dass Menschen ihre irrigen Standpunkte im Anschluss umso vehementer vertreten. Wie die zuvor angeführten Effekte ist auch der Backfire-Effekt hinlänglich aus Offline-Diskussionen bekannt und keineswegs speziell an Social-Media-Diskurse gebunden. So wurden Personen mit starken Vorurteilen gegenüber der Grippeimpfung in einer US-amerikanischen Studie noch skeptischer hinsichtlich der Impfung, nachdem man sie mit Sachargumenten des zuständigen Centers for Disease Control and Prevention versorgt hatte (Nyhan und Reifler 2015). In vergleichbaren Social-Media-Settings suchen Menschen offenbar verstärkt die Rückendeckung ihrer jeweiligen Commu${ }^{18} \mathrm{https}: / /$ de-de.facebook.com/aerztegegentierversuche, 8. September 2017 -Zugegriffen: 09. September
2017. Hervorhebungen von C. K. 
nitys, nachdem sie mit Gegenargumenten zur eigenen Position konfrontiert werden. So wandten sich Facebook-Nutzer, die Verschwörungsmythen anhängen, ebenso wie solche, die regelmäßig auf redaktionell geprüfte Informationen positiv reagieren, teils noch stärker ihrer jeweiligen Echokammer Gleichgesinnter zu, nachdem sie Beiträge zu lesen bekamen, die ihre eigene Ansicht in Frage stellten. Die Anhänger der Verschwörungsmythen beschäftigen sich dabei allerdings kaum mit Korrekturen, und ihre Sympathiebekundungs- und Kommentarraten für weitere Verschwörungsbeiträge stiegen nach der kontrollierten Interaktion sogar noch (Zollo et al. 2017).

Gruppenpolarisierung $\mathrm{Ob}$ offline am Stammtisch oder online in einem sozialen Netzwerk: Diskussionsteilnehmerinnen und -teilnehmer äußern sich bevorzugt entlang einer bereits vorherrschenden Stoßrichtung. Die Folge: Gleichlautende Argumente werden wiederholt im Gedächtnis aktiviert und so leichter wieder abrufbar. Nicht nur bei den Sprechern, sondern auch bei den Zuhörern festigen sich vorhandene Einstellungen auf diese Weise weiter. Häufig verschärfen Diskutanten in Debatten zudem den Ton, um mehr Aufmerksamkeit zu erzielen - mit der Folge, dass manche Teilnehmerinnen und Teilnehmer nach Gruppendiskussionen zu extremeren Ansichten neigen als zuvor. Dieses in sozialen Netzwerken häufig zu beobachtende Phänomen wird durch (vermeintliche oder tatsächliche) Nichtverfolgbarkeit weiter verstärkt, denn anonyme Online-Diskussionen führen zu besonders extremen Meinungen (Sia et al. 2002). Zur Veranschaulichung des Effekts mögen die Kommentare zweier Nutzerinnen auf der Facebook-Seite von Spektrum der Wissenschaft unter einem dort geposteten homöopathiekritischen Artikel dienen:

Die Schulmedizin hat mehr Denkfehler! Gesunde Zellen zerstören um gesund zu werden?? (Chemo...bringt nichts) Die echte Homöopathie ist nicht Zucker! ${ }^{19}$

Der folgende Kommentar wurde rund 10 Stunden später eingestellt:

Verreckt an Eurer Pharmascheisse. Tierversuche, schwere Nebenwirkungen und null Heilung. Hauptsache, Geld fliesst in die Taschen der Pharma. Euer Denkfehler ist es, daß es ewig so weitergeht. ${ }^{20}$

\footnotetext{
${ }^{19}$ https://de-de.facebook.com/spektrumverlag, 7. September 2017 - Zugegriffen: 08. September 2017. ${ }^{20} \mathrm{Ebd}$.
} 
Emotional Contagion Dieser auch unter "Gefühlsansteckung“ bekannte Effekt besagt, dass sich Menschen in Gruppendiskussionen an den affektiven Zustand ihrer Peers angleichen. So ist bekannt, dass Facebook-Nutzer nach dem Lesen trauriger oder fröhlicher Kommentare ihrer Freunde selbst häufiger Beiträge in entsprechender Tonlage posten. Dabei verbreiten sich Inhalte, die wütend machen, online besonders erfolgreich (Berger und Milkman 2012).

False News Value Effekt Dieser Effekt wird hier im Sinne eines Vorschlags neu eingeführt. Er besagt, dass Falschmeldungen ein besonders dynamisches Ausbreitungsverhalten (in sozialen Medien, vermutlich aber auch ganz allgemein) zeigen, weil die Teilenden mit ihnen ihr soziales Ansehen stärker steigern können als mit (längst) validierten und (bekanntermaßen) wahren Aussagen. Der Effekt beschreibt damit die Kombination zweier getrennter Phänomene: Zum einen heben Menschen ihr soziales Ansehen, ihre „social currency", wenn sie wichtige oder überraschende Informationen mit anderen teilen (Berger 2016). Zum anderen teilen sie (in sozialen Medien) Falschmeldungen schneller und häufiger als validierte Nachrichten, wie bereits für Twitter gezeigt wurde (Vosoughi et al. 2018). Unser natürliches Verhalten befördert demnach die Verbreitung von Falschmeldungen, weil diese im Schnitt überraschender erscheinen als wahre. ${ }^{21}$

Die beschriebenen psychologischen Effekte sind unter den Bedingungen sozialer Netzwerke - so die These - im Schnitt wirkmächtiger als in vergleichbaren Situationen der Auswahl und Bereitstellung von Information und Meinung in Offline-Kontexten. Mit anderen Worten: Bereits vorhandene Auffassungen, bestehende Ressentiments und politische Einstellungen manifestieren sich durch Diskurse in sozialen Netzwerken tendenziell stärker, als dies infolge von Offline-Diskussionen der Fall ist, wo die Effekte jedoch gleichfalls auftreten und für alle Menschen - bei individuellen Unterschieden, die vor allem in Persönlichkeitseigenschaften wurzeln dürften - typisch sind. Auf gesellschaftlicher Ebene ist die Folge eine stärkere Polarisierung und Fragmentierung. Und auch bezüglich Sachfragen wird die Gesellschaft unter der Wirkung sozialer Medien gespaltener.

Die Tatsache, dass dieselben psychologischen Mechanismen unter SocialMedia-Bedingungen im Schnitt stärkere Wirkungen provozieren, liegt u. a. in technologischen Effekten begründet, wie sie charakteristisch für

\footnotetext{
${ }^{21} \mathrm{Da}$ dieser Effekt hier neu vorgeschlagen wird, ist er - anders als die anderen angeführten Effekte nicht bereits offline erforscht. Es erscheint freilich plausibel, dass er auch unabhängig von digitalen oder sozialen Medien virulent ist - dies jedoch wäre erst noch empirisch zu überprüfen.
} 


\begin{tabular}{|c|c|c|c|c|}
\hline Effekt & Umschreibung & $\begin{array}{l}\text { Offline- } \\
\text { Kontexte }\end{array}$ & $\begin{array}{l}\text { Social } \\
\text { Media }\end{array}$ & $\begin{array}{l}\text { Wirkung } \\
\text { verstärkt durch }\end{array}$ \\
\hline Confirmation Bias & $\begin{array}{l}\text { Bevorzugung von Informationen, } \\
\text { die uns bestätigen }\end{array}$ & + & ++ & \multirow{4}{*}{$\begin{array}{l}\text { - Filterbubble-Effekt } \\
\text { - Echokammer-Effekt } \\
\text { - Quantifizierung und } \\
\text { scheinbare } \\
\text { Objektivierung des } \\
\text { Selbstwerts durch }\end{array}$} \\
\hline Partisan Bias & $\begin{array}{l}\text { Informationen der Gegenseite } \\
\text { werden abgewertet }\end{array}$ & + & ++ & \\
\hline Motivated Reasoning & $\begin{array}{l}\text { Im engeren Sinne: Vermeidung } \\
\text { kognitiver Dissonanz bei } \\
\text { Entscheidungen }\end{array}$ & + & ++ & \\
\hline Backfire-Effekt & $\begin{array}{l}\text { Entgegengebrachte Sachargumente } \\
\text { führen zu mehr Widerstand }\end{array}$ & + & ++ & \\
\hline Gruppenpolarisierung & $\begin{array}{l}\text { Diskussionsteilnehmer äußern } \\
\text { sich entlang vorherrschender } \\
\text { Stoßrichtung }\end{array}$ & + & ++ & \multirow{3}{*}{$\begin{array}{l}\text { Metriken von Likes, } \\
\text { Fans, Retweets usw. } \\
\text { - Anonymität (faktisch } \\
\text { oder vermeintlich) } \\
\text { - Social Bots }\end{array}$} \\
\hline Emotional Contagion & $\begin{array}{l}\text { Angleichung an den affektiven } \\
\text { Zustand der Peers }\end{array}$ & + & ++ & \\
\hline False News Value Effekt & $\begin{array}{l}\text { Falsche Nachrichten sind wertvoller, } \\
\text { da meist überraschender }\end{array}$ & $(+)$ & ++ & \\
\hline
\end{tabular}

Abb. 7 Verschiedene psychologische Effekte tragen dazu bei, dass sich Einstellungen zu (kontroversen) Themen im Zuge der Auswahl und des Teilens von Informations- und Meinungsbeiträgen tendenziell erhärten. Diese Effekte sind auch in Offline-Kontexten virulent, können aber unter den Bedingungen sozialer Netzwerke im Durchschnitt stärkere Wirkungen entfalten. Das hat verschiedene, insbesondere auch technologische Gründe. So fördern die auf Big-Data-Auswertungen durch KI-Systeme basierenden, individuell auf jeden Nutzer zugeschnittenen Interaktionsangebote sozialer Netzwerke Filterbubble- und Echokammer-Effekte. Auch die für soziale Medien charakteristischen Favorisierungsmetriken wie Anzahl von Likes („Gefällt mir"), Followern und Retweets, die (faktische oder vermeintliche) Anonymität in Online-Diskussionen sowie gezielte Manipulation durch automatisierte Diskussionsteilnehmer, sogenannte Social Bots, können zur Verstärkung der Effekte beitragen. (Quelle: Autor)

soziale Netzwerke sind (Abb. 7). Dies sei beispielhaft anhand der Gruppenpolarisierung erläutert. Für die (tatsächliche oder lediglich angenommene) Anonymität in Online-Diskussionen wurde eine verschärfte Gruppenpolarisierung bereits nachgewiesen (Sia et al. 2002). Auch der Umstand, dass der Selbstwert von Nutzern durch die Anzahl ihrer Likes, Follower oder Retweets scheinbar objektiviert wird und Nutzer mit ihren Diskussionsbeiträgen somit in direkte kommunikative Konkurrenz zueinander treten, dürfte Gruppenpolarisierung in sozialen Medien fördern; insbesondere emotionalisierende Äußerungen regen zu stärkerer Interaktion an, werden häufiger favorisiert, geteilt und kommentiert. Da die in sozialen Netzwerken zugestellten Informationen und Meinungsäußerungen seitens der Plattformanbieter vollautomatisch daraufhin ausgewählt werden, jede Nutzerin und jeden Nutzer mit größtmöglicher Wahrscheinlichkeit zur erneuten Interaktion auf der Plattform zu veranlassen, fördern auch Filterbubble-Effekte die weitere Polarisierung innerhalb der Echokammern. Des Weiteren dürfte 
ein etwaiges Eingreifen von Social Bots Gruppenpolarisierungseffekte verstärken, sofern die Bots daraufhin programmiert sind, Argumente einseitig zu penetrieren und die Tonlage dabei ggf. noch zu verschärfen.

Zwischenfazit Mehrere in der Mehrzahl gut dokumentierte psychologische Effekte, denen wir alle beim Auswählen und Teilen von Informationen und Meinungen mehr oder weniger stark unterliegen, können zu einer Erhärtung vorhandener Einstellungen führen. Unter den Bedingungen sozialer Netzwerke entfalten die Effekte im Durchschnitt stärkere Wirkungen als in vergleichbaren Offline-Settings. Damit können soziale Netzwerke als eine Ursache steigender gesellschaftlicher Polarisierung und zunehmender Fragmentierung von Öffentlichkeit angesehen werden. Um die zugrunde liegenden Phänomene noch genauer zu fassen und der erheblichen gesellschaftlichen Bedeutung des Phänomens besser gerecht zu werden, müssen technologische, psychologische, soziologische, politik-, medien- und kommunikationswissenschaftliche sowie fachdidaktische Forschungsperspektiven künftig stärker integriert werden.

\section{Vertrauen in die Wissenschaft und Akteure in sozialen Medien}

Auch wenn sich vorhandene wissenschaftsskeptische Einstellungen unter den Gegebenheiten sozialer Netzwerke eher manifestieren können, bedeutet das nicht automatisch, dass sich immer mehr Personen entsprechende Positionen zu eigen machen, dass sich also durch die Nutzung sozialer Medien per se Wissenschaftsskepsis ausbreitet. Tatsächlich gibt es keine Hinweise auf eine aktuell wachsende Wissenschaftsskepsis in Deutschland. Laut dem bevölkerungsrepräsentativen Wissenschaftsbarometer 2018 (Wissenschaft im Dialog 2018a) vertrauen 54 Prozent der Deutschen Wissenschaft und Forschung eher oder voll und ganz; 39 Prozent sind diesbezüglich unentschieden; 7 Prozent vertrauen Wissenschaft und Forschung nicht oder eher nicht. Die Zahlen ähneln denen des Vorjahrs: 2017 äußerten 50 Prozent Vertrauen und 12 Prozent Misstrauen; 37 Prozent zeigten sich unentschieden. Ebenfalls stabil sind zentrale Einzelbefunde: Jüngere äußern mehr Vertrauen, Ältere mehr Misstrauen als der Bevölkerungsschnitt; höhere Bildung geht mit mehr Vertrauen einher; Wähler von AfD und Linke äußern im Schnitt mehr Misstrauen $^{22}$ (Wissenschaft im Dialog 2018b; vgl. Könneker 2018).

\footnotetext{
${ }^{22}$ Jeweils am Ende der Telefonbefragungen des Wissenschaftsbarometers wird die „Sonntagsfrage“ gestellt.
} 
Vertrauen in Experten entsteht einem psychologischen Modell zufolge, wenn Nicht-Experten ihnen Können/Expertise, Integrität und gute Absichten/Wohlwollen zuschreiben (Mayer et al. 1995; Hendriks et al. 2015). Wissenschaftler erhalten demnach positive Vertrauensurteile, wenn ihre Vertrauensgeber davon ausgehen, dass sie erstens über die notwendige Expertise zur Erkenntnisgewinnung und Problemlösung verfügen, zweitens sich in der eigenen Forschungsarbeit an begründbare Methoden und die Regeln ihrer jeweiligen Fachdisziplin halten und drittens durch ihre Forschung Nutzen für andere oder die Allgemeinheit mehren wollen. Die Daten des Wissenschaftsbarometers geben starke Hinweise darauf, dass Misstrauen gegenüber Wissenschaft hierzulande eher selten aufgrund (mutmaßlich) mangelnder Expertise entsteht, sondern weit häufiger wegen (mutmaßlich) unzureichender Integrität oder (mutmaßlich) zweifelhafter Beweggründe der Forschenden sowie speziell durch fragwürdige Abhängigkeiten, in denen diese (mutmaßlich) stehen. Daraus lässt sich folgern, dass die praktische externe Wissenschaftskommunikation stärker auch die Methodik und sogar die Leerstellen von Forschung wie auch die persönlichen Motive der Forschenden explizit machen sollte (Könneker 2018); wertvoll sollten hier Ich-Botschaften der Forschenden selbst sein, da diese von Rezipienten und Dialogpartnern als besonders authentisch aufgefasst werden dürften. Dabei schadet es der Reputation der Wissenschaftlerinnen und Wissenschaftler vermutlich auch nicht, wenn Rezipienten feststellen, dass sich Forschende untereinander auch kritisieren - insbesondere dann nicht, wenn sie bereit sind, eigene Fehler einzuräumen (Hendriks et al. 2016).

Für eine derart gestaltete Wissenschaftskommunikation bieten gerade soziale Netzwerke prinzipiell gute Voraussetzungen (Könneker und Lugger 2013): Zum einen sind deren Zutrittsbarrieren - anders als bei journalistischen Massenmedien - gering, zum anderen sind Ich-Botschaften in sozialen Medien gebräuchlich und attraktiv. Darüber hinaus wäre mehr fachliche Expertise in Social-Media-Diskursen, authentisch eingebracht von Wissenschaftlerinnen und Wissenschaftlern selbst, auch aus gesellschaftlicher Perspektive wünschenswert. Denn bezüglich der Frage, wer hierzulande die Social-Media-Diskurse zu Wissenschaftsthemen prägt, sind dem Wissenschaftsbarometer 2018 starke Hinweise darauf zu entnehmen, dass es keineswegs ein Querschnitt der Gesellschaft ist, der hier aktiv ist. Demnach scheinen insbesondere AfD-Nahe - und damit tendenziell wissenschaftsskeptischer als der Bevölkerungsschnitt eingestellte Personen - die Diskurse über Wissenschaftsthemen in sozialen Netzwerken zu formen. 33 Prozent der Deutschen informieren sich laut Wissenschaftsbarometer 2018 (auch) in sozialen Netzwerken wie Facebook oder Twitter 
über Wissenschaft und Forschung - nicht eingerechnet sind hier Blogs und Videoportale wie Youtube. Doch unter den nur 5 Prozent, die in sozialen Netzwerken wie Facebook oder Twitter häufig oder sehr häufig Informationen oder Meinungen über Wissenschaft und Forschung selbst posten und somit Diskurse anstoßen, sind AfD-Nahe wahrscheinlich deutlich überrepräsentiert. Dasselbe gilt sowohl für jene 6 Prozent, die Posts zu Wissenschaftsthemen liken oder favorisieren, als auch für jene lediglich 2 Prozent, die diese in Social Media kommentieren (Könneker 2018).

Parteien aus dem populistischen rechten Spektrum stellen nach Ansicht von Beobachtern eine Herausforderung für die Freiheit der Wissenschaft dar, was Erfahrungen in verschiedenen europäischen Nationen belegen, wo sie Regierungsbeteiligung erlangt haben (Nature 2018). Wenn SocialMedia-Diskurse zu Wissenschaftsthemen stark von Personen - und hier wahrscheinlich größtenteils von Neuen Akteuren, die sich an populistischen Strömungen oder Parteien orientieren oder solchen anhängen - geprägt werden, berührt das demzufolge gerade auch die Wissenschaft selbst.

Wissenschaftlerinnen und Wissenschaftler aus Deutschland bringen sich im Vergleich zu Fachkollegen aus anderen Nationen offenbar deutlich seltener mit ihrer fachlichen Expertise in Social-Media-Diskurse ein. Das legt eine Befragungsstudie mit international rekrutierten Nachwuchsforschenden, Teilnehmerinnen und Teilnehmern der Lindauer Nobelpreisträgertagungen bzw. des Heidelberg Laureate Forums, nahe (Könneker et al. 2018). So engagierten sich innerhalb eines Zweijahreszeitraums vor ihrer Befragung nur 24,6 Prozent der in den letzten Jahren vor allem oder ausschließlich in Deutschland forschenden bis 35-Jährigen mit ihrer fachlichen Expertise in sozialen Netzwerken wie Facebook oder Twitter; ihre Kollegen aus anderen europäischen Ländern taten dies zu 32,1 Prozent, Forschende aus den USA zu 40,7 Prozent und solche aus asiatischen Ländern gar zu 52,3 Prozent. Entsprechende Unterschiede bestehen beim Erstellen und Hochladen von Webvideos über die eigene Forschung sowie beim Wissenschaftsbloggen (Abb. 8).

Die Studie liefert zudem mögliche Gründe für die auch bei den meisten Offline-Formaten der externen Wissenschaftskommunikation vorhandene relative Zurückhaltung hiesiger Forscherinnen und Forscher. Zum einen meinen signifikant weniger von ihnen, dass sich ein Engagement in externer Wissenschaftskommunikation für eine Karriere innerhalb der Wissenschaft auszahle. Einer entsprechenden Aussage stimmten nur 51,3 Prozent der 224 befragten hiesigen Nachwuchsforschenden zu. Ihre 744 Kollegen aus anderen Nationen bejahten dies im Schnitt hingegen zu 74,2 Prozent, wobei die Zustimmung bei den in den USA Forschenden am stärksten war (Abb. 9). 
$\% 60$
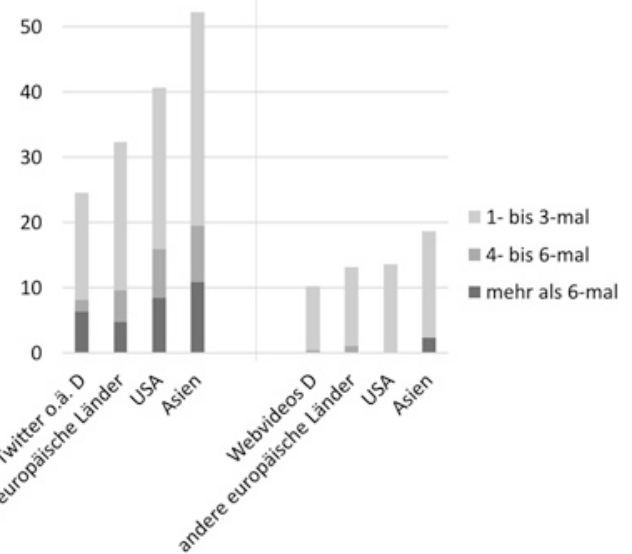

$\% \quad 30$
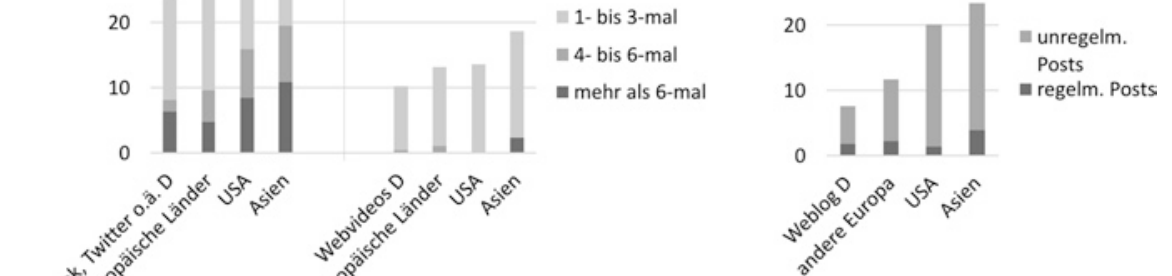

Abb. 8 Selbst genutzte Social-Media-Formate junger Forscherinnen und Forscher für die eigene externe Wissenschaftskommunikation ( $N=988$; Befragte Deutschland $(D)=224$, andere europäische Länder $=272$, USA $=214$, Asien $=128$ ). (Quelle: Autor)

»Wissenschaftskommunikation hat einen positiven Einfluss auf eine Karriere in der Wissenschaft.«

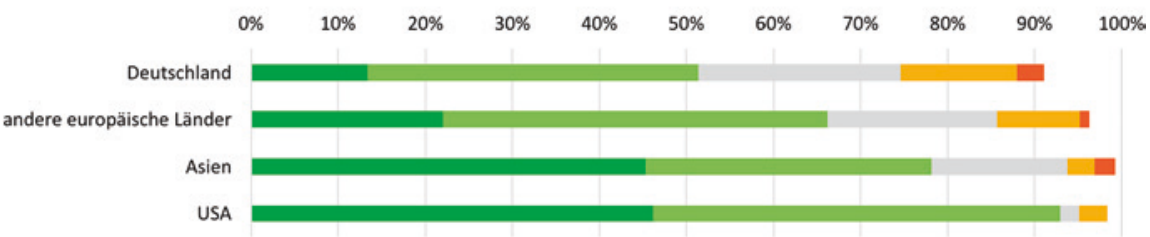

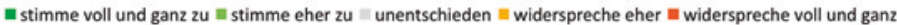

Abb. 9 Hiesige junge Forschende der MINT-Disziplinen sowie der Wirtschaftswissenschaften sind im Vergleich zu ihren Kolleginnen und Kollegen in anderen Ländern deutlich skeptischer bezüglich der Frage, ob sich ein Engagement in externer Wissenschaftskommunikation für eine Karriere innerhalb der Wissenschaft auszahlt ( $N=988$; Befragte Deutschland $=224$, andere europäische Länder $=272$, Asien $=128$, USA $=214$ ). (Quelle: Autor)

Zum anderen werden künftige Forschende in anderen Ländern offenbar systematischer auf eigene Beiträge zur externen Wissenschaftskommunikation vorbereitet. So beurteilten die in Deutschland forschenden Jungwissenschaftlerinnen und -wissenschaftler ihre Möglichkeiten, bereits während des Studiums praktische Fertigkeiten in der Vermittlung von Wissenschaft zu erwerben, retrospektiv zu 80,3 Prozent als schlecht oder sehr schlecht und nur 
wManche Hochschulen bieten ihren Studierenden praxisorientierte Schulungen in der Vermittlung wissenschaftlicher Themen an Nicht-Wissenschaftler an. Wenn Sie an Ihr eigenes Studium zurückdenken, wie beurteilen Sie die Möglichkeiten, sich entsprechende Fähigkeiten anzueignen?«

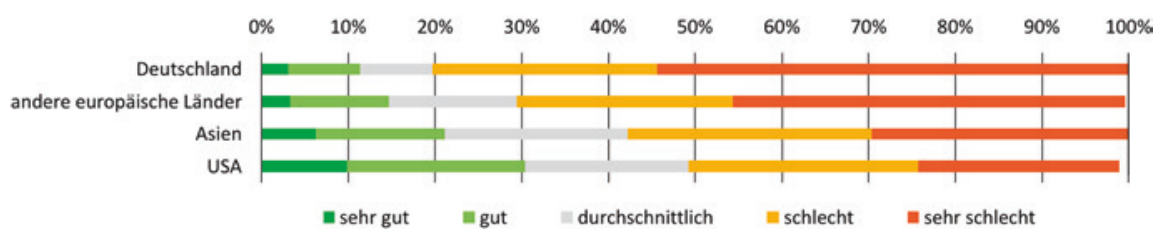

Abb. 10 Im Vergleich zu ihren Kolleginnen und Kollegen, die in anderen Ländern forschen, beurteilen hiesige junge Forschende der MINT-Disziplinen sowie der Wirtschaftswissenschaften die Möglichkeiten, im Studium in praktischer Wissenschaftskommunikation ausgebildet zu werden, retrospektiv deutlich schlechter $(\mathrm{N}=988$; Befragte Deutschland $=224$, andere europäische Länder $=272$, Asien $=128$, USA = 214). (Quelle: Autor)

zu 11,4 Prozent als gut oder sehr gut. Damit zeichnen sie ein noch düstereres Bild als ihre Kolleginnen und Kollegen in anderen Ländern Europas oder Asien. Die besten Möglichkeiten, sich während des Studiums auf die Praxis der externen Wissenschaftskommunikation vorzubereiten, dürften demnach junge Forschende in den USA haben, die ihre entsprechenden Möglichkeiten rückschauend zu 30,6 Prozent als gut oder sehr gut beurteilten (Abb. 10).

Mehr Anerkennung für persönliches Engagement in externer Wissenschaftskommunikation in den wissenschaftlichen Institutionen und mehr Schulungsangebote, um Hintergrundwissen über den Medienbetrieb sowie praktische Fertigkeiten zu erwerben, erscheinen daher als wichtige Maßnahmen auf dem Weg zu einer Verbesserung der Situation.

Gleichwohl sollte man sich nicht der Illusion hingeben, sämtlichen Herausforderungen einer „post-faktischen“ Mediengesellschaft allein durch intensivere, verbesserte praktische Wissenschaftskommunikation von Forschenden begegnen zu können. Darauf machen Lewandowsky, Ecker und Cook mit ihrem Technocognition-Ansatz aufmerksam (Lewandowsky et al. 2017a, b). Sie schließen ebenfalls, dass ein komplexes Zusammenspiel psychologischer und technologischer Ursachen bewirkt, dass sich Fehlinformationen in digitalen Diskursen hochgradig dynamisch und mit besonders nachhaltigen Folgen verbreiten. Adäquate Antworten darauf sollten folglich in erster Linie technologische Maßnahmen unter Beachtung psychologischer Prinzipien darstellen. Konkret schlagen sie etwa die Ausweitung algorithmischer Filterbubble-Radien vor mit dem Ziel, dass auch Inhalte aus anderen Echokammern in den individualisierten Newsfeeds sozialer Netzwerke berücksichtigt werden, oder die Entwicklung von Factchecking-Tools, die falsche Informationen 
automatisch identifizieren und kennzeichnen. Diese könnten auf SocialMedia-Plattformen integriert werden, um Nutzer vor der eigenen Interaktion auf fehlerhafte Informationen aufmerksam zu machen.

Unter dem Strich jedoch sei die „post-trouth malaise“ keine Folge von Kommunikationsversäumnissen, sondern eine gesellschaftliche, ja politische Krise mit technologischen und psychologischen Versatzstücken. Gezielt gestreute Fehlinformationen und eine dadurch geschürte Wissenschaftsskepsis stellen demzufolge rationale Strategien zur Verfolgung politischer und ökonomischer Ziele dar. Um die Krise zu meistern, bedürfe es folglich $\mathrm{zu}$ allererst eines dezidierten politischen Willens. Vor diesem Hintergrund erscheint eine quantitativ wie qualitativ verbesserte externe Wissenschaftskommunikation in sozialen Medien nur als eine, wenn auch notwendige Bedingung dafür, wissenschaftsbezogene Social-Media-Diskurse performanter zu gestalten und negative Auswirkungen auf die Wissenschaft und ihre gesellschaftliche Verankerung zu verhindern.

Trotz dieser Einschränkung steht in diesem Sinne zu wünschen, dass mehr Forschende die sozialen Medien mit deren Tendenz, vorhandene Einstellungen und Vorurteile zu zementieren, in der eigenen externen Wissenschaftskommunikation berücksichtigen. ${ }^{23}$ Es geht um die Glaubwürdigkeit von Wissenschaft, um die Bereitstellung verlässlicher Expertise, authentischer Einsichten und methodischen Know-hows für eine demokratisch-pluralistische Gesellschaft, die in steigendem Ausmaß soziale Netzwerke zur Informations- und Meinungsbeschaffung auch zu Wissenschaftsthemen nutzt. Wo entsprechende inhaltliche Angebote in Social Media schlicht nicht zur Verfügung stehen, können auch politische und technologische Maßnahmen Desinformation und Wissenschaftsskepsis kaum eindämmen.

\section{Literatur}

Acatech, et al. (Hrsg.). (2017). Social Media und digitale Wissenschaftskommunikation. Analyse und Empfehlungen zum Umgang mit Chancen und Risiken in der Demokratie. München: Acatech.

Allgaier, J. (2018). Science and medicine on youtube. In J. Hunsinger, L. Klastrup, \& M. M. Allen (Hrsg.), Second international handbook on internet research (S. 1-21). Dordrecht: Springer.

\footnotetext{
${ }^{23}$ Schäfer (2016) macht in seiner Analyse von disziplinenübergreifenden Befragungen ebenfalls darauf aufmerksam, dass Forschende sowie Institutionen Onlinemedien weniger stark für externe Wissenschaftskommunikation nutzen als andere Akteursgruppen. Sofern sie es doch tun, stehen als wichtigste Motive Unterrichtung der Öffentlichkeit sowie Führen gesellschaftlicher Dialoge im Vordergrund.
} 
Allgaier, J. (2019). Cool geeks, dangerous nerds, entrepreneurial scientists and idealistic physicians? Exploring science and medicine in popular culture. In A. Görgen, G. A. Nunez, \& H. Fangerau (Hrsg.), Handbook of popular culture and biomedicine (S. 25-39). Cham: Springer.

Berger, J. (2016). Contagious: Why things catch on. New York: Simon \& Schuster.

Berger, J., \& Milkman, K. L. (2012). What makes online content viral? Journal of Marketing Research, 49, 192-205.

Del Vicario, M., et al. (2016). The spreading of misinformation online. Proceedings of the National Academy of Sciences, 113(3), 554-559.

Dogruel, L., \& Beck, K. (2017). Social Media als Alternative der Wissenschaftskommunikation? Eine medienökonomische Analyse. In P. Weingart, et al. (Hrsg.), Perspektiven der Wissenschaftskommunikation im digitalen Zeitalter (S. 123-187). Weilerswist: Velbrück.

Edelman Trust Barometer. (2018). Global report. https://www.edelman.com/ sites/g/files/aatuss191/files/2018-10/2018_Edelman_Trust_Barometer_Global_ Report_FEB.pdf. Zugegriffen: 19. Nov. 2018.

Frees, B., \& Koch, W. (2018). ARD/ZDF-Onlinestudie 2018: Zuwachs bei medialer Internetnutzung und Kommunikation. Media Perspektiven, 9, 398-413. http://www.ard-zdf-onlinestudie.de/ardzdf-onlinestudie-2018/artikel-2018/.

Frimer, J. A., Skitka, L. J., \& Motyl, M. (2017). Liberals and conservatives are similarly motivated to avoid exposure to one another's opinions. Journal of Experimental Social Psychology, 72, 1-12.

Hagenhoff, S., et al. (2007). Neue Formen der Wissenschaftskommunikation. Eine Fallstudienuntersuchung. Göttingen: Universitätsverlag.

Hendriks, F., Kienhues, D., \& Bromme, R. (2015). Measuring laypeople's trust in experts in a digital age: The Muenster Epistemic Trustworthiness Inventory (METI). PLoS ONE, 10(10), e0139309.

Hendriks, F., Kienhues, D., \& Bromme, R. (2016). Disclose your flaws! Admission positively affects the perceived trustworthiness of an expert science blogger. Studies in Communication Sciences, 16(2), 124-131.

Könneker, C. (2016). Wissenschaftskommunikation in vernetzten Öffentlichkeiten. In H. Bonfadelli, et al. (Hrsg.), Forschungsfeld Wissenschaftskommunikation (S. 453-476). Wiesbaden: Springer VS.

Könneker, C. (2018). Vertrauen, Misstrauen, Social Media - Schlüsse aus dem Wissenschaftsbarometer 2018. https://www.wissenschaftskommunikation.de/ vertrauen-misstrauen-social-media-schluesse-aus-dem-wissenschaftsbarometer-2018-19243. Zugegriffen: 19. Nov. 2018.

Könneker, C., \& Lugger, B. (2013). Public Science 2.0 - Back to the future. Science, 342(6154), 49-50.

Könneker, C., Niemann, P., \& Böhmert, C. (2018). Weniger Wertschätzung, weniger Engagement. Zur Situation der Wissenschaftskommunikation in Deutschland. Forschung und Lehre, 10, 870-872.

Kunda, Z. (1990). The case of motivated reasoning. Psychological Bulletin, 108(3), 480-498. 
Lewandowsky, S., Ecker, U. K. H., \& Cook, J. (2017a). Beyond misinformation: Understanding and coping with the "post-truth" era. Journal of Applied Research in Memory and Cognition, 6, 353-369.

Lewandowsky, S., Cook, J., \& Ecker, U. K. H. (2017b). Letting the gorilla emerge from the mist: Getting past post-truth. Journal of Applied Research in Memory and Cognition, 6, 418-424.

Mayer, R. C., Davis, J. H., \& Schoorman, F. D. (1995). An integrative model of organizational trust. The Academy of Management Review, 20(3), 709-734.

Nature. (2018). Beware the rise of the radical right. Nature, 563, 599 (Editorial).

Neuberger, C. (2014). Social Media in der Wissenschaftsöffentlichkeit. Forschungsstand und Empfehlungen. In P. Weingart \& P. Schulz (Hrsg.), Wissen - Nachricht - Sensation. Zur Kommunikation zwischen Wissenschaft, Öffentlichkeit und Medien (S. 315-368). Weilerswist: Velbrück.

Nyhan, B., \& Reifler, J. (2015). Does correcting myths about the flu vaccine work? An experimental evaluation of the effects of corrective information. Vaccine, 33(3), 459-464.

Özelt, C. (2018). Literatur im Jahrhundert der Physik. Geschichte und Funktion interaktiver Gattungen 1900-1975. Göttingen: Wallstein.

Schäfer, M. (2016). Wissenschaftskommunikation Online. In B. Heinz, et al. (Hrsg.), Forschungsfeld Wissenschaftskommunikation (S. 275-293). Wiesbaden: Springer VS.

Schäfer, M. (2017). Wissenschaftskommunikation ist Wissenschaftsjournalismus, Wissenschafts-PR ... und mehr. https://www.wissenschaftskommunikation. de/wissenschaftskommunikation-ist-wissenschaftsjournalismus-wissenschafts-pr-und-mehr-3337. Zugegriffen: 31. Okt. 2018.

Sia, C.-L., Tan, B. C. Y., \& Wei, K.-K. (2002). Group polarization and computer-mediated communication: Effects of communication cues, social presence, and anonymity. Information Systems Research, 13(1), 70-90.

Vosoughi, S., Roy, D., \& Aral, S. (2018). The spread of true and false news online. Science, 359(6380), 1146-1151.

Wissenschaft im Dialog. (Hrsg.). (2018a). Wissenschaftsbarometer 2018. Berlin: WiD. https://www.wissenschaft-im-dialog.de/projekte/wissenschaftsbarometer/ wissenschaftsbarometer-2018/.

Wissenschaft im Dialog. (Hrsg.). (2018b). Wissenschaftsbarometer 2018: Ergebnisse nach Subgruppen. Berlin: WiD. https://www.wissenschaft-im-dialog.de/projekte/ wissenschaftsbarometer/wissenschaftsbarometer-2018/.

Zollo, F., et al. (2017). Debunking in a world of tribes. PLoS ONE, 12(7), e0181821.

Carsten Könneker ist Chefredakteur von Spektrum.de und Spektrum der Wissenschaft. Von 2012 bis 2018 bekleidete er den Lehrstuhl für Wissenschaftskommunikation und Wissenschaftsforschung am Karlsruher Institut für Technologie und war am Aufbau des dortigen Studiengangs „Wissenschaft - Medien - Kommunikation“ beteiligt. Von 2012 bis 2015 leitete er zudem als Gründungsdirektor das Nationale Institut für Wissenschaftskommunikation (NaWik). 
Open Access Dieses Kapitel wird unter der Creative Commons Namensnennung 4.0 International Lizenz (http://creativecommons.org/licenses/by/4.0/deed.de) veröffentlicht, welche die Nutzung, Vervielfältigung, Bearbeitung, Verbreitung und Wiedergabe in jeglichem Medium und Format erlaubt, sofern Sie den/die ursprünglichen Autor(en) und die Quelle ordnungsgemäß nennen, einen Link zur Creative Commons Lizenz beifügen und angeben, ob Änderungen vorgenommen wurden.

Die in diesem Kapitel enthaltenen Bilder und sonstiges Drittmaterial unterliegen ebenfalls der genannten Creative Commons Lizenz, sofern sich aus der Abbildungslegende nichts anderes ergibt. Sofern das betreffende Material nicht unter der genannten Creative Commons Lizenz steht und die betreffende Handlung nicht nach gesetzlichen Vorschriften erlaubt ist, ist für die oben aufgeführten Weiterverwendungen des Materials die Einwilligung des jeweiligen Rechteinhabers einzuholen. 